\title{
Clinical evaluation of microendoscopy-assisted extreme lateral interbody fusion
}

\author{
Tomohide Segawa ${ }^{1}$, Hirohiko Inanami ${ }^{1}$, Hisashi Koga ${ }^{2}$ \\ ${ }^{1}$ Department of Orthopaedic Surgery, Inanami Spine and Joint Hospital, Tokyo, Japan; ${ }^{2}$ Department of Orthopaedics, Iwai Orthopaedic Medical \\ Hospital, Tokyo , Japan \\ Contributions: (I) Conception and design: H Inanami; (II) Administrative support: T Segawa; (III) Provision of study materials or patients: H Inanami; \\ (IV) Collection and assembly of data: T Segawa; (V) Data analysis and interpretation: T Segawa, H Koga; (VI) Manuscript writing: All authors; (VII) \\ Final approval of manuscript: All authors. \\ Correspondence to: Hisashi Koga, MD, PhD. Deputy Director, the Head of Education and Training Center, Department of Orthopaedics, Iwai \\ Orthopaedic Medical Hospital, Tokyo, Japan. Email: hkoga0808@gmail.com.
}

Background: Extreme lateral interbody fusion (XLIF) has rapidly become a popular operative procedure for lumbar canal stenosis and scoliosis. This approach provides direct access to the lateral aspect of the disc with minimal disruption of spinal structures, including ligaments and muscles. However, it involves risk of injuries to the lumbar nerve plexus, segmental artery and intestinal tract because of the limited surgical field. This study aimed to clarify the benefit of using a microendoscope to prevent these injuries.

Methods: A total of 96 consecutive patients treated by a single surgeon were retrospectively analyzed. The basic approach via the psoas muscle was performed in accordance with the conventional XLIF procedure. Operative manipulations, such as insertion of the shim, discectomy, endplate preparation and intervertebral spacer placement, were performed with the assistance of a microendoscope. Preoperative and postoperative neurological status were evaluated using the Japanese Orthopedic Association (JOA) scores and the Oswestry Disability Index (ODI).

Results: Eighty-four patients underwent surgery for a single vertebral level, nine patients underwent surgery for 2 levels and three patients underwent surgery for 3 levels (average, 1.2 levels). The average age of patients was 61 years (range, 22-83 years); the mean follow-up period was 18 months (range, 3-36 months). Average preoperative and postoperative JOA scores were 11.9 and 15.6, with a mean recovery rate of $33 \%$. Average preoperative and postoperative ODI scores were 38.6 and 19.1. There were $3(3.1 \%)$ complications: 2 end-plate fractures and 1 deep surgical site infection. There were no bowel perforations or vascular injuries.

Conclusions: Microendoscopy assistance is one solution for severe visceral and vascular injuries related to XLIF.

Keywords: Microendoscope; endoscope; extreme lateral interbody fusion; extreme lateral interbody fusion (XLIF); complication

Submitted Jul 08, 2017. Accepted for publication Jul 17, 2017.

doi: $10.21037 /$ jss.2017.08.09

View this article at: http://dx.doi.org/10.21037/jss.2017.08.09

\section{Introduction}

Extreme lateral interbody fusion (XLIF; NuVasive Inc., San Diego, CA, USA) is a minimally invasive technique for the treatment of lumbar canal stenosis and scoliosis. XLIF has become a popular surgical technique and an alternative method for conventional anterior and posterior approaches for interbody fusion (1). This approach provides direct access to the lateral aspect of the vertebral disc with minimal disruption of the surrounding structures, including ligaments and muscles. A large cage can be inserted in the intervertebral space without disrupting the anterior and 

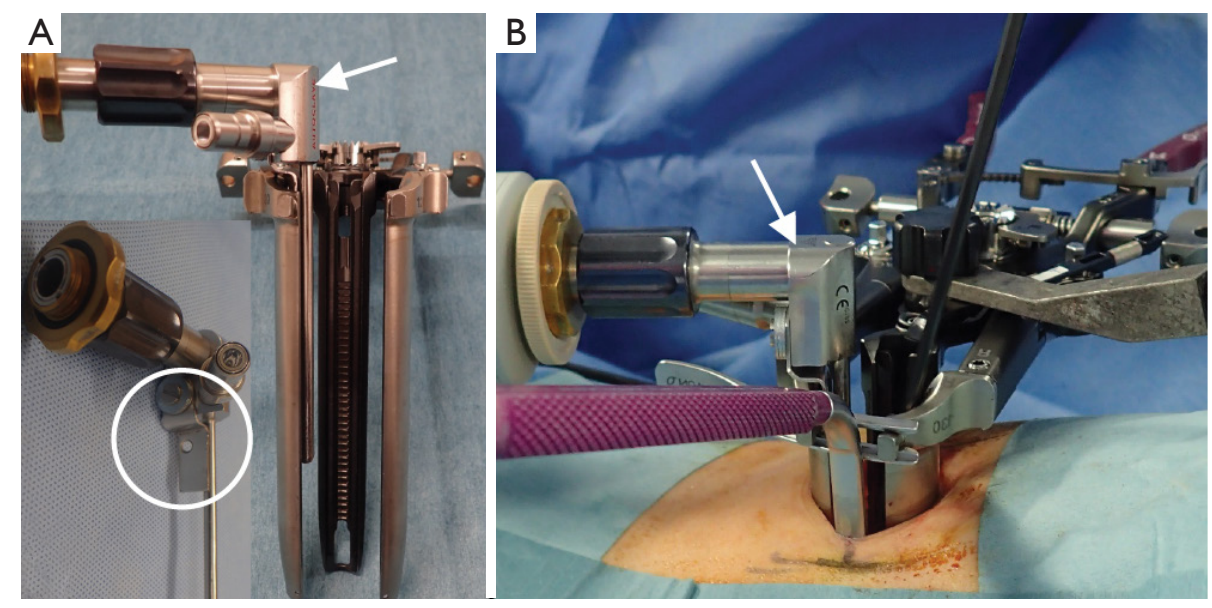

Figure 1 A microendoscope with a custom-ordered attachment and intraoperative view. (A) A microendoscope is attached inside the Maxcess 4 retractor; (B) intraoperative view. White arrows indicate the camera head and white circle indicates the custom-ordered attachment.

posterior annulus fibrosis or longitudinal ligaments with minimal risk to the retroperitoneal structures and the great vessels $(2,3)$.

However, some major complications are associated with the lateral approach, such as lumbar nerve plexus injury, segmental arterial injury and bowel perforation (4-6). Among these complications, segmental arterial injury and bowel perforation are fatal, and only a few reports regarding how to avoid these complications are available (6-9). These complications are troublesome because it is very difficult to find these complications during surgery owing to the narrow surgical space and the limited visual field.

To overcome these difficulties, we have been using a microendoscope for operative assistance since XLIF was introduced in our hospital. This study aimed to prove the superiority of microendoscopy-assisted XLIF compared to the conventional XLIF procedure.

This retrospective study analyzed the first consecutive 96 patients treated with microendoscopy-assisted XLIF between April 2013 and July 2016 by a single surgeon (Inanami H). Data regarding age, sex, levels of interbody fusion, estimated blood loss (EBL) and surgical time were collected from medical records. Operative and anesthesia records were also reviewed. The mean followup period was 18 months (range, 3-36 months), and major complications were identified. Preoperative and postoperative neurological status were evaluated using the Japanese Orthopedic Association (JOA) scores and Oswestry Disability Index (ODI).

\section{Methods}

\section{Surgical procedure}

The XLIF approach has been described in detail previously (1). Surgery was performed with the patient in the lateral decubitus position (left side up). The operating table was slightly bent at the iliac region to enlarge the intervertebral space on the approach side. An approximately 2 -cm longitudinal incision was made to accommodate the surgeon's index finger, which was inserted anteriorly through the muscle layers to identify the retroperitoneal space. After passing through the fascia and accessing the retroperitoneal space, the index finger was used to sweep the peritoneum anteriorly and then to palpate down to the psoas muscle. Once the psoas muscle was identified, the index finger was swept up to the direct lateral target mark. An incision was made at this direct lateral location and an initial dilator was introduced. Sequential dilation was performed under evoked electromyography in directional orientations with discrete threshold responses (NV M $5^{\circledR}$; $\mathrm{NuVasive}$ Inc.) to provide information regarding the location of the lumbar nerve plexus during access to the lateral aspect of the disc. Following placement of the third dilator, an expandable retractor (Maxcess $4^{\circledR}$; NuVasive Inc.) was placed over the final dilator. Finally, a microendoscope (Medtronic Sofamor Danke, Memphis, TN, USA) was attached to an expandable retractor with a custom-ordered attachment (made by Inanami H; Figure 1).

After installation of a microendoscope, we first confirmed that the retractor existed in the retroperitoneal 
space. With the combination of direct visualization using the microendoscope and electromyogram monitoring, we confirmed that the lumbar nerve plexus and the branches of the segmental artery were not in the surgical field. After that, the shim was safely inserted in the posterior borders of the corresponding vertebral disc and an anterior retractor was inserted in the ventral side of the anterior longitudinal ligament (ALL) (Figure 2). Subsequently, the clearly visualized lateral part of the annulus fibrosis was incised and discectomy and interbody implant placement were performed (Figure 3). After placement completion, the retractor was removed slowly to observe the psoas muscle rebounding and to confirm there was no excessive bleeding

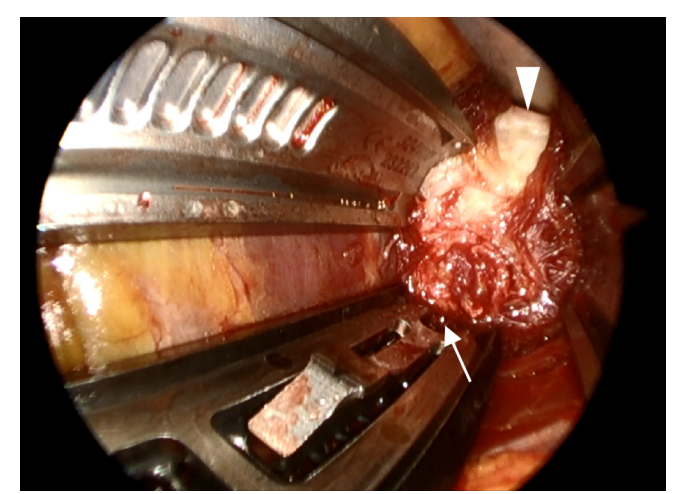

Figure 2 Intraoperative microendoscopic view of the retroperitoneal space. Right: Cranial view. Left: Caudal view. White arrow indicates the shim and white arrowhead indicates anterior longitudinal ligament (ALL). or visceral injury.

\section{Results}

Forty-one men and 55 women with an average age of 61 years (range, 22-83 years) underwent this procedure. The mean follow-up period was 18 months (range, 3-36 months). The most common primary diagnosis was spondylolisthesis (42 patients; $43.8 \%$ ), followed by degenerative disc disease (35 patients; $36.5 \%$ ) and spinal canal stenosis (10 patients; 10.4\%). Eighty-four procedures involved a single vertebral level, nine involved two levels and three involved three levels (average, 1.2 levels) (Table 1). Supplemental posterior instrumentation was used for all patients.

The mean operative time per level required for the anterior procedure was 47 minutes (range, 21-109 minutes) and mean estimated intraoperative blood loss per level was $38 \mathrm{~mL}$ (range, $1-110 \mathrm{~mL}$ ). The average preoperative and postoperative JOA scores were 11.9 and 15.6, with a mean recovery rate of $33 \%$. The average preoperative and postoperative ODI scores were 38.6 and 19.1 (Table 2).

There were $3(3.1 \%)$ major complications. Two complications were L4 and L5 end-plate fractures that improved with conservative treatment. One complication was a deep surgical site infection that was diagnosed at 2 months after surgery. Reoperation was performed as follows: after only the cage was removed, debridement and then bone grafting were performed in the intervertebral disc space. Surgical site infection was resolved with antibiotic
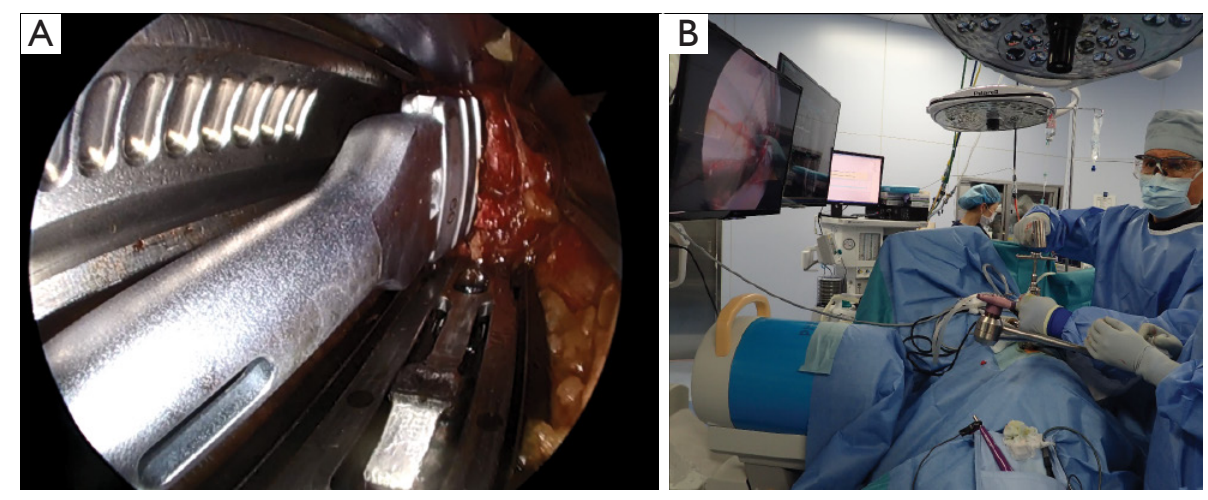

Figure 3 Intraoperative microendoscopic view (A) and operator's standing position and visual point (B) during an interbody implant placement. Note that surgical instruments do not interfere with the field (A) and the operator has a good and comfortable posture (B). 
Table 1 Patient characteristics

\begin{tabular}{lc}
\hline Characters & Value \\
\hline Age (years) & $61 \pm 12.7$ \\
Sex (male:female) & $41: 55$ \\
Primary diagnosis & \\
Spondylolisthesis & 42 \\
Degenerative disc disease & 35 \\
Spinal canal stenosis & 10 \\
Degenerative scoliosis & 5 \\
Others & 4 \\
Number of fused levels per patient & 1.2 \\
\hline
\end{tabular}

Table 2 Patient outcomes

\begin{tabular}{lc}
\hline Variables & Value \\
\hline Operative time per level (min) & $47 \pm 19.0$ \\
Blood loss per level $(\mathrm{mL})$ & $38 \pm 30.6$ \\
JOA score & \\
Pre & $11.9 \pm 4.5$ \\
Post & $15.6 \pm 5.4$ \\
Improvement rate (\%) & 33 \\
ODI & \\
Pre & $38.6 \pm 12.6$ \\
Post & $19.1 \pm 16.6$ \\
\hline
\end{tabular}

JOA, Japanese Orthopedic Association; ODI, Oswestry Disability Index.

treatment for 3 months. There were no bowel perforations or vascular injuries.

\section{Discussion}

Microendoscopy-assisted spine surgery is a widespread minimally invasive technique in Japan (10). This technique was first applied to lumbar disc herniation (11). Then, it was extended to spinal canal decompression and interbody fusion (12). However, to our knowledge, XLIF has not been performed using a microendoscope. Therefore, we applied the microendoscope to the disc manipulation stage of the XLIF procedure.
The microendoscopic technique has two major advantages. First, a microendoscopic lens is angled at $25^{\circ}$ and the viewpoint of a microendoscope exists in the body during surgery. Therefore, visualization of the lateral aspect is superior to what can be achieved with the unaided eye or surgical loupes, which has a viewpoint outside the body. It has been reported that approximately $25 \%$ of segmental artery branches at the L4 and L5 vertebral bodies existed behind the lateral aspect of the vertebral disc, that is, in the insertion position of the shim (13). Even though it is difficult to observe insertion of the shim with the unaided eye or surgical loupes, this microendoscopic technique makes it possible to insert the shim while observing the surgical field (Figure 2). Secondly, the surgeon, assistant and scrub nurse can all check the same surgical field through the microendoscopic view. As a result, it is possible to accurately ascertain the progress of surgery so that it can proceed smoothly.

However, this technique also has disadvantages such as a steep learning curve and the need for training under expert surgeons. In particular, it is important to be proficient in bidimensional surgery using a microendoscope. Ebata et al. reported the importance of preoperative training for microendoscopic surgery (14). They stated that if it is possible to receive appropriate and efficient guidance from expert surgeons, then the learning curve can be shortened.

In 2011, Rodgers et al. performed the largest study of complications during 600 XLIF procedures and reported that overall incidence of complications was $6.2 \%$ (4). Youssef et al. reviewed 14 XLIF reports by various authors and reported major complication rates were 4-8.6\% (15). Our major complication rates were less than those of previous reports.

The limitation of this study was the lack of a control group for comparison. Although further studies are necessary, we speculate that major complications will not increase by using a microendoscope because this technique is not a new concept. It is a modification of established methods.

\section{Conclusions}

Despite the steep learning curve and the need for training under expert surgeons, microendoscopy-assisted XLIF is a potential alternative to the conventional XLIF procedure because of its efficacy and safety. 


\section{Acknowledgements}

None.

\section{Footnote}

Conflicts of Interest: The authors have no conflicts of interest to declare.

Ethical Statement: This study was approved by ethics committee of the Iwai Medical Foundation, and informed consent was obtained from the patients for publication of this study and any accompanying images.

\section{References}

1. Ozgur BM, Aryan HE, Pimenta L, et al. Extreme lateral interbody fusion (XLIF): a novel surgical technique for anterior lumbar interbody fusion. Spine J 2006;6:435-43.

2. Shamji MF, Isaacs RE. Anterior-only approaches to scoliosis. Neurosurgery 2008;63:139-48.

3. Sharma AK, Kepler CK, Girardi FP, et al. Lateral lumbar interbody fusion: clinical and radiography outcomes at 1 year: a preliminary report. J Spinal Disord Tech 2011;24:242-50.

4. Rodgers WB, Gerber EJ, Patterson J. Intraoperative and early postoperative complications in extreme lateral interbody fusion: an analysis of 600 cases. Spine (Phila Pa 1976) 2011;36: 26-32.

5. Assina R, Majmundar NJ, Herschman Y, et al. First report of major vascular injury due to lateral transpsoas approach leading to fatality. J Neurosurg Spine 2014;21:794-8.

6. Balsano M, Carlucci S, Ose M, et al. A case report of a

Cite this article as: Segawa T, Inanami H, Koga H. Clinical evaluation of microendoscopy-assisted extreme lateral interbody fusion. J Spine Surg 2017;3(3):398-402 . doi: 10.21037/ jss.2017.08.09 rare complication of bowel perforation in extreme lateral interbody fusion. Eur Spine J 2015;24 Suppl 3:405-8.

7. Sakai T, Tezuka F, Wada K, et al. Risk management for avoidance of major vascular injury due to lateral transpsoas approach. Spine (Phila Pa 1976) 2016;41:450-3.

8. Takata Y, Sakai T, Tezuka F, et al. Risk assessment of lumbar segmental artery injury during lateral transpsoas approach in the patients with lumbar scoliosis. Spine (Phila Pa 1976) 2016;41: 880-4.

9. Deukmedjian AR, Le TV, Dakwar E, et al. Movement of abdominal structures on magnetic resonance imaging during positioning changes related to lateral lumbar spine surgery: a morphometric study. J Neurosurg Spine 2012;16:615-23.

10. Yoshimoto $M$, Iwase $T$, Takebayashi $T$, et al. Microendoscopic discectomy for far lateral lumbar disk herniation: less surgical invasiveness and minimum 2-year follow-up results. J Spinal Disord Tech 2014;27:E1-7.

11. Foley KT, Smith MM. Microendoscopic discectomy. Tech Neurosurg 1997;3:301-7.

12. Isaacs RE, Podichetty VK, Santiago P, et al. Minimally invasive microendoscopy-assisted transforaminal lumbar interbody fusion with instrumentation. J Neurosurg Spine 2005;3:98-105.

13. Tezuka F, Sakai T, Nishisho T, et al. Variation in arterial supply to the lower lumbar spine. Eur Spine J 2016;25:4181-7.

14. Ebata S, Ooba T, Orii H, et al. Learning curve in endoscopic surgeries: A comparative study among seven surgeons. J Spine Res 2012;3:1117-21.

15. Youssef JA, Mcafee PC, Patty CA, et al. Minimally invasive surgery: lateral approach interbody fusion: results and review. Spine (Phila Pa 1976) 2010;35:S302-11. 